\title{
Les systèmes pathogènes complexes. modélisation et simulation
}

\author{
Éric Daudé
}

\section{(2) OpenEdition}

Journals

Édition électronique

URL : http://journals.openedition.org/rfst/601

DOI : $10.4000 /$ rfst.601

ISSN : 2492-3672

\section{Éditeur}

Espaces et SOciétés (UMR 6590)

\section{Référence électronique}

Éric Daudé, «Les systèmes pathogènes complexes. modélisation et simulation », Revue francophone sur la santé et les territoires [En ligne], Ateliers et séminaires, mis en ligne le 25 novembre 2016, consulté le 06 avril 2021. URL : http://journals.openedition.org/rfst/601 ; DOI : https://doi.org/ $10.4000 /$ rfst.601

Ce document a été généré automatiquement le 6 avril 2021.

\section{c) (i) (2)}

La Revue francophone sur la santé et les territoires est mise à disposition selon les termes de la Licence Creative Commons Attribution - Pas d'Utilisation Commerciale - Partage dans les Mêmes Conditions 4.0 International. 


\title{
Les systèmes pathogènes complexes. modélisation et simulation
}

\author{
Éric Daudé
}

1 Dans la lignée des travaux des géographes sur les complexes pathogènes (M. Sorre, 1933) et les systèmes pathogènes ( $H$. Picheral, 1983), nous présentons ici quelques exemples destinés à promouvoir le concept de système pathogène complexe. En effet, dans le vocabulaire des sciences de la complexité, les risques pour la santé résultent moins d'une succession d'évènements que d'une articulation complexe de facteurs (Daudé, Vaguet \& Paul, 2015).

2 Prenons l'épidémie de grippe de 2017 : La surmortalité qui sera probablement mesurée en France est-elle dû à une réduction de la couverture vaccinale ? À un vaccin moins efficace ou une grippe plus virulente ? À une médecine de ville et des hôpitaux publics surchargés ? À des conditions climatiques exceptionnelles? La réponse est ardue car les épidémies s'inscrivent dans des systèmes socio-spatiaux hétérogènes, constitués de nombreux niveaux décisionnels aux motivations et priorités diverses. Il en va ainsi des composants et comportements individuels, influencés par les structures familiales et les normes sociales, elles-mêmes rythmées selon les politiques de santé publiques et les contextes sociaux, qui sont en partie le reflet des forces du marché et des changements environnementaux. Ces systèmes comportent donc de nombreux éléments qui ont leurs propres dynamiques, sont évolutifs et en interactions permanentes. Ceux-ci produisent cette variabilité sociale et géographique de l'expression des épidémies, que l'on ne saurait réduire à de simples causalités. Pris ensemble, ces différents composants sont les termes classiques des systèmes pathogènes complexes. De tels systèmes possèdent des propriétés qui peuvent constituer des opportunités d'intervention en santé publique.

\section{Le charme discret des systèmes complexes}

3 Un système pathogène complexe est un système ouvert. C'est ce que l'on observe lorsque le virus Ébola sort de son aire d'endémie via les mobilités des populations 
(Gomes et al., 2014), ou lorsque des pratiques culturelles se "propagent»: l'alimentation fast-food, et induisent de nouveaux risques, l'obésité (De Vogli et al., 2014). Si cette ouverture du système accroit le risque de diffusion de pathogènes ou de pratiques à risque, elle oblige également à une meilleure coopération internationale en matière de santé, et notamment de surveillance et de prévention.

4 Un système pathogène complexe présente des dynamiques non-linéaires. Dans les maladies à vecteurs (paludisme, dengue, Zika...), les densités vectorielles sont certes liées aux conditions climatiques mais aussi aux politiques de contrôle et aux variations spatiales des gites de ponte (Reiter, 2008). De petits évènements volontaires (destructions ciblées de gites) ou des variations incontrôlées (climatique) pourraient alors générer, par effet systémique, de grand effets (réduction ou extension des aires de présence du vecteur). La recherche de ces seuils et « petits évènements » à l'origine de grands impacts devrait devenir un objectif prioritaire dans la lutte contre les épidémies.

5 Un système pathogène complexe est caractérisé par une variabilité spatio-temporelle de l'information. L'omniprésence des flux d'information dans ces systèmes assure la réactivité de ses éléments face aux changements: la diffusion des pratiques de prévention a été un moyen de lutte efficace contre le sida (Hornik, 2002). Cette propriété peut donc être mise au service de la santé publique. C'est le cas par exemple des études sur les mots-clés (liés à la grippe, au choléra etc.) des requêtes sur Internet ou dans les réseaux sociaux qui servent à suivre la progression d'une maladie dans des pays où le système de surveillance est défaillant (Chunara et al., 2012). Mais ces flux peuvent être sources d'influence interdépendantes et contradictoires: amalgames et contre-vérités scientifiques, crédibilités des opérateurs publics et lobbying de l'industrie pharmaceutique seraient à confronter aux évolutions de la couverture vaccinale.

6 Un système pathogène complexe connaît des processus d'auto-organisation. Ces processus décentralisés génèrent spontanément des propriétés nouvelles et organisées, sans l'intervention d'une autorité supérieure. Ces processus sont à l'origine de l'émergence des patients-experts du fait de changements dans la relation au corps et de l'avènement de la société de l'information (Boudier et al., 2012). Ces phénomènes émergents, ou innovations sociales, peuvent parfois être "récupérés » par le système pour s'imposer à l'ensemble, notamment s'ils engendrent de meilleures performances ou s'ils permettent une réduction des dépenses publiques (Donaldson, 2003).

7 Un système pathogène complexe présente une sensibilité aux conditions initiales, son évolution est en partie liée à son histoire. Les états, expériences et expositions passés ont donc une part importante dans la dynamique à venir du système. La séroprévalence est un exemple classique de cette sensibilité, la propagation d'un virus dans une population étant en partie liée à ses précédents passages et au taux d'immunité acquise par la population (Ooi et al., 2006).

8 Enfin un système pathogène complexe est toujours loin de l'équilibre, en équilibre instable. Si l'équilibre est considéré dans le cas des maladies vectorielles non pas comme l'absence de la maladie mais comme la circulation du virus à bas bruit, alors cet équilibre peut basculer vers des bouffées épidémiques, voire des pandémies, du fait des propriétés dont nous venons de parler: ouverture, interactions locales et globales, dynamiques non-linéaires, sensibilité aux conditions initiales. Les allergies ou les 
déficiences immunitaires sont d'autres exemples, à l'échelle de l'individu, de l'équilibre instable du système immunitaire.

\section{Apprendre en modélisant à base d'agents}

Si ces concepts issus des sciences de la complexité sont élégants, pour mieux caractériser une définition déjà ancienne d'un système pathogène (Picheral, 1983; Handschumacher \& Hervouët, 2004); nos pratiques de recherche nous conduisent à les formaliser par des modèles et à explorer leurs dynamiques par la simulation informatique, apportant ainsi l'élément manquant au système pathogène complexe (Gatrell, 2005). La modélisation à base d'agents constitue le cadre méthodologique employé. Techniquement il s'agit de systèmes informatiques composés d'entités appelées agents, qui interagissent mutuellement ou avec leur environnement, qui possèdent le plus souvent un but ou un objectif à atteindre et qui procèdent selon des modes de coopération, de concurrence ou de coexistence. Ce qu'il faut retenir ici c'est la souplesse de ce type de modélisation algorithmique, basé sur des propriétés et des méthodes appliquées à des agents, par rapport à une modélisation mathématique basé sur des fonctions et des processus appliquées à des variables.

Les modèles à base d'agents permettent en effet d'intégrer dans un même système une grande variété de catégories qui agissent et une forte hétérogénéité entre les individus de ces catégories. On peut ainsi modéliser des hôtes, des vecteurs et un système de soins, avec des différenciations sociodémographiques, génétiques et géographiques. La prise en compte de cette diversité interindividuelle peut s'avérer intéressante dans la recherche d'effets macroscopiques d'interventions réalisées sur des populations cibles.

Les modèles à base d'agents offrent également la possibilité de formaliser explicitement des comportements, comme les mobilités individuelles, et des mécanismes décisionnels, comme le choix d'une pratique de prévention individuelle. Le choix de tel ou tel comportement peut s'appuyer sur des informations qui sont inégalement distribuées dans l'espace, le temps et entre agents. Ces comportements peuvent par ailleurs être contradictoires ou évolutifs selon les informations reçues et ce faisant être plus proche des pratiques observées, telles que les dynamiques d'opinion par exemple.

12 Les modèles à base d'agents donnent enfin à l'espace un statut de composant du système modélisé. Il peut donc être décrit comme un agent, avec les hétérogénéités et les dynamiques géographiques et environnementales jugées nécessaires à l'évolution du système. La construction d'un modèle épidémiologique à l'échelle d'une ville intégrera ainsi les modes d'utilisation du sol, les densités de population ou encore la localisation des hôpitaux, en général toute information spatiale supposée agir dans le système ainsi décrit.

\section{Le cas exemplatif de $\mathrm{MO}^{3}$}

13 Illustrons maintenant ces concepts et options méthodologiques avec le projet $\mathrm{MO}^{3}$. Les maladies à transmissions vectorielles (MTV) sont un enjeu majeur de santé publique avec un fort potentiel d'explosions épidémiques, au regard notamment du changement climatique et des dynamiques d'urbanisation. La surveillance et le contrôle de ces MTV passent notamment par la production de bases de données géographiques et de 
modèles dynamiques. Ceux-ci peuvent contribuer à l'évaluation des risques vectoriels et des risques épidémiques à différentes échelles, notamment micro-locale des espaces urbains. Dans ce contexte, $\mathrm{MO}^{3}$ est un simulateur de maladies à transmission vectorielle dont l'objectif est d'explorer la capacité de reproduction et de dispersion du vecteur Aedes aegypti selon différentes configurations géographiques et le rôle des mobilités humaines dans la dispersion des virus aux échelles intra-urbaine.

$\mathrm{MO}^{3}$ est un modèle à base d'agents où sont décrits les quatre principaux sous-système de la dengue : les humains (Cebeillac \& Daudé, 2016), les moustiques (Maneerat \& Daudé, 2016), l'environnement (Misslin \& Daudé, 2016) et les virus. Bangkok (Thaïlande) et Delhi (Inde) sont les métropoles d'étude sélectionnées pour calibrer ces différents composants.

Tout d'abord la population d'hôtes est générée informatiquement de façon à respecter statistiquement le genre et la pyramide des âges, observée dans la réalité à une échelle fine. Pour chaque agent des attributs concernant le statut immunitaire sont créés, en relation avec la base de données sur les virus, afin de suivre la progression de la maladie. Des données issues de l'analyse de réseaux sociaux (Twitter) permettent de calibrer une partie des agendas des mobilités individuelles. Dans le jargon de la modélisation agents, l'ensemble de cette phase consiste à construire une population synthétique, socialement et spatialement représentative de la population cible.

16 Le vecteur est modélisé à l'échelle de l'individu pour les adultes et sous la forme de stocks de moustiques pour les stades aquatiques. Si le formalisme employé pour modéliser les stocks et leurs transitions repose sur des fonctions mathématiques, la modélisation des comportements du moustique individualisé s'appuie sur les connaissances entomologiques, notamment en termes de prédation, de ponte et de conquête territoriale.

17 Enfin l'environnement est défini à la fois selon les besoins et les contraintes liées au cycle de vie du moustique et aux activités humaines. Le modèle environnemental intègre donc des caractéristiques hétérogènes dans le type d'espace (usages du sol), dans le temps (variations climatiques) et dans les comportements (contrôle différencié des gites de pontes).

18 Le couplage de ces trois sous-systèmes avec un quatrième module représentant une base de connaissance sur les virus constitue donc le modèle $\mathrm{MO}^{3}$. La construction de ce modèle participe donc d'une part à une démarche heuristique de formalisation des connaissances autour d'un système pathogène complexe et d'autre part à un besoin d'explorer des dynamiques endémies-épidémies selon différents scénarii. Ainsi les villes «synthétiques» seront destinées à accueillir des exercices de simulations d'hypothèses de recherche et à mesurer des dynamiques qu'il n'est pas possible d'observer dans la réalité, tels que les lieux de contamination par les moustiques.

Concluons cette présentation rapide en apportant quelques réponses à des critiques qui sont régulièrement adressées à l'encontre de cette catégorie de modèles à base d'agents (comportemental et individu-centré) par rapport à des modèles mathématiques (de fonction et agrégé).

20 La première critique porte sur l'intérêt limité, voire contre-productif, d'une modélisation à une échelle aussi fine, là où des propriétés agrégées suffisent à caractériser des dynamiques macroscopiques. Signalons que de telles propriétés macroscopiques ont pour la plupart été révélées par l'observation directe ou par une 
multitude d'expérimentations, qui sont extrêmement compliquées à mettre en œuvre en épidémiologie spatiale, ou qui, lorsqu'elles existent, sont sujettes aux nombreux biais connus des données de surveillance. En partant des comportements, du moustique et de l'hôte par exemple, on intègre des connaissances qui reposent là aussi sur de nombreuses études (déplacement, espace de vie, prédation) pour ensuite produire, par la simulation, des connaissances macroscopiques qui peuvent non seulement converger vers des observations faites par ailleurs (par exemple sur la capacité de dispersion de cohortes de moustiques) mais aussi vers des connaissances nouvelles difficiles à observer sur le terrain (graphe des cycles de contamination hôtes-vecteurs par exemple). Le fait de prendre en compte la diversité des types d'agents, leur hétérogénéité et leurs comportements permet ainsi l'exploration d'une très grande variété de dynamiques.

21 La seconde critique, liée en partie à la première, porte sur la difficulté, voire l'impossibilité, de calibrer, de valider et d'interpréter les résultats issus des simulations de modèles intégrant de nombreux composants et interactions non-linéaires. C'est effectivement un verrou que tout modélisateur prend en compte lorsqu'il réalise ce type de modèle dans lequel il peut $\mathrm{y}$ avoir des dizaines voire des centaines de paramètres. Tout d'abord, la calibration du modèle repose pour l'essentiel sur les connaissances dans les différents domaines d'exercice du modèle (en entomologie par exemple). Chaque valeur de paramètre doit donc reposer sur des données dont les plages de variation sont connues afin de réduire au maximum l'incertitude. Ensuite on dispose aujourd'hui de méthodes statistiques liés à l'explosion des big-data qui permettent de traiter et d'analyser des volumes de données tels que ceux en sortie de simulations. Enfin il reste la question de la validation, qu'il faut "désacraliser » par rapport aux pratiques de validation classique. Il est en effet toujours possible, même si le nombre de paramètre libre n'est pas très grand, de faire converger les simulations vers des valeurs empiriques observées. Il faut donc d'une part s'assurer que cette convergence se réalise dans le respect des plages de variations connues des paramètres des entités élémentaires. Il faut d'autre part discuter avec les experts du domaine des diverses dynamiques du modèle. Certes une même situation peut-être reproduite à partir de différentes combinaisons de paramètres, mais n'est-ce pas là une possibilité du monde réel! Il faut enfin mettre à la disposition de tous ces modèles et jeux de données pour faciliter la reproductibilité des simulations.

Pour conclure, il y a donc des avantages à construire des modèles à base d'agents, spatialisés et explicites, pour l'épidémiologie des systèmes pathogènes complexes. Les hypothèses à l'origine de chacun des comportements modélisés et de leurs conditions de mise en œuvre sont aisément testables et discutables. Leur modélisation et l'étude de leurs dynamiques durant la simulation permettent de mesurer leur contribution dans l'évolution du système, et éventuellement de les supprimer si le système se comporte de manière aberrante par rapport à nos connaissances. Plutôt que de modéliser des processus définis à partir d'observations macroscopiques, la modélisation de comportements microscopiques peut permettre de faire émerger des comportements surprenants. Ceux-ci poseront de nouvelles questions et orienteront vers des études de terrain, pour confirmer ou infirmer ces solutions. En matière de modélisation et de simulation à base d'agents en épidémiologie spatiale, je prônerai donc de l'ambition pour les programmes de recherche et de la modestie dans les conclusions tirées des résultats des simulations. 


\section{BIBLIOGRAPHIE}

Boudier F., Bensebaa F., Jablanczy A. (2012), L'émergence du patient-expert : une perturbation innovante. Innovations, 3, 39:13-25.

Cebeillac A., Daudé É. (2016), Où et Quand ? Que nous apprennent les données Twitter et Google Map sur les mobilités quotidiennes de Bangkok (Thaillande). $15^{\mathrm{e}}$ coll. Mobilités Spatiales, Fluidité Sociale (MSFS), Champs-sur-Marne, https://msfs2016.sciencesconf.org/111848

Chunara R., Andrews J., Brownstein J. (2012), Social and News Media Enable Estimation of Epidemiological Patterns Early in the 2010 Haitian Cholera Outbreak. The American Society of Tropical Medicine and Hygiene, 86, 1: 39-45.

Daudé É., Vaguet A., Paul R. (2015), La dengue, maladie complexe. Natures Sciences Sociétés, 23, 4: 331-342, DOI:10.1051/nss/2015058

De Vogli R., Kouvonen A., Gimeno D. (2014), The influence of market deregulation on fast food consumption and body mass index: a cross-national time series analysis. Bulletin of World Health Organisation, 92:99-107.

Donaldson L. (2003), Expert patients usher in a new era of opportunity for the NHS. British Medical Journal, 326, 7402: 1279-1280.

Gatrell A. (2005), Complexity theory and geographies of health: a critical assessment. Social Science \& Medicine, 60;12:2661-71.

Gomes M., Pastore y Piontti A., Rossi L., Chao D., Longini I., Halloran M., Vespignani A. (2014), Assessing the International Spreading Risk Associated with the 2014 West African Ebola Outbreak. PLOS Currents Outbreaks.

Handschumacher P., Hervouët J.-P. (2004), Des systèmes pathogènes à la santé publique : une nouvelle dimension pour la géographie de la santé tropicale, Autrepart, 1;29:47-63.

Hornik R. (Ed.). (2002), Public health communication: Evidence for behavior change. Routledge, London, p. 421.

Maneerat S., Daudé É. (2016), A spatial agent-based simulation model of the dengue vector Aedes aegypti to explore its population dynamics in urban areas. Ecological Modelling, 333:66-78, 10.1016/ j.ecolmodel.2016.04.012.

Misslin R., Daudé É. (2016), Génération d'environnements artificiels pour la simulation spatiale d'arboviroses en milieu urbain : application à la dengue et au virus Zika. $16^{\mathrm{e}}$ coll. SAGEO, Nice, https://sageo2016.sciencesconf.org/

Ooi E., Goh K., Gubler D. (2006), Dengue prevention and 35 years of vector control in Singapore. Emerging Infectious Diseases, 12;6:887-93.

Picheral H. (1983), Complexes et systèmes pathogènes : approche géographique, De l'épidémiologie à la géographie humaine. Travaux et documents de géographie tropicale, $\mathrm{n}^{\circ} 48$, p. 5-22.

Reiter P. (2008), Global warming and malaria: knowing the horse before hitching the cart. Malaria Journal, 7:Suppl 1, S3. 
INDEX

Mots-clés : système pathogène complexe, modélisation, maladies à transmissions vectorielles

AUTEUR

ÉRIC DAUDÉ

CNRS, UMR IDEES 